\title{
Boundary Integral Equations Method for the Time-Harmonic Electromagnetic Scattering \\ Kuo-Ming Lee*
}

Department of Mathematics, National Cheng Kung University, Taiwan

\begin{abstract}
In contrast to the classical boundary layer approach which is not uniquely solvable at the irregular wave numbers, the modified layer method always has a unique solution to the exterior boundary problem for the vector Helmholtz equation. We compare the stability of the two methods numerically.
\end{abstract}

Keywords: Boundary integral equation; Electromagnetic scattering; Helmholtz equation

\section{Introduction}

Consider the exterior boundary value problem for the scalar Helmholtz equation in $\mathrm{R}^{\mathrm{n}}, \mathrm{n}=2,3$

$$
\Delta \mathrm{u}+\mathrm{k}^{2} \mathrm{u}=0, \mathrm{k} \in \mathrm{R}, \mathrm{k} \neq 0 \text {, in } \mathrm{R}^{\mathrm{n}} \backslash \mathrm{D}
$$

with prescribed Dirichlet or Neumann boundary condition. For the uniqueness of this problem, usually the asymptotic behavior of the solution at infinity will also be given. Using the classical boundary layer approach (see $[1,2])$, this kind of problem can be solved uniquely using the double layer potential for the Dirichlet boundary condition, the single layer potential for the Neumann boundary condition, respectively, provided $k$ is not an interior eigenvalue of the $-\Delta$ operator (see [3]). Since physically the uniqueness of the exterior boundary value problem is not in question, the cause of the non-uniqueness must have come from our mathematical method. Remaining in the framework of boundary integral equation method, various modifications have been developed to over-come this non-uniqueness for all $k \neq 0$ ([4] by adding a volume potential, [5-7] by using the combined boundary layers).

In $[8,9]$, the method of combined layers has been modified slightly and extended to the case of a vector Helmholtz equation. In this paper, we compare the numerical results of the classical boundary integral equation methods with those of the modified boundary integral equation methods with combined boundary layers for the vector Helmholtz equation. The plan of this paper is as follows. In the second section, we deduce the exterior boundary value problem from the time harmonic electromagnetic scattering problem from an ideal conductor. In the third section, we use the modified boundary layers approach to win a system of Fredholm integral equation of the second kind which is uniquely solvable. A section on numerical method is then followed by another giving some numerical examples. At the end of the paper, we give some properties of the system of the boundary integral equations from section 3 in the appendix.

\section{Boundary Value Problem}

Consider the electromagnetic scattering from a homogeneous, isotropic obstacle in $\mathrm{R}^{3}$. The electric Field $\varepsilon$ and the magnetic Field' $\mathrm{H}$ satisfy the Maxwell equations

$$
\operatorname{rot} \varepsilon+\mu \frac{\partial H}{\partial t}=0, \quad \operatorname{rot} H-\mu \frac{\partial \varepsilon}{\partial t}=\sigma \varepsilon,
$$

Where $\varepsilon$ is the dielectricity, $\mu$ is the permeability and $\sigma$ is the conductivity of the medium. For the time-harmonic electromagnetic wave of the form

$$
\begin{aligned}
& \varepsilon(x, t)=\operatorname{Re}\left\{\left(\in+\frac{i \sigma}{\omega}\right)^{-1 / 2} E(x) e^{-i \omega t}\right\}, \\
& H(x, t)=\operatorname{Re}\left\{\left(\epsilon+\frac{i \sigma}{\omega}\right)^{-1 / 2} E(x) e^{-i \omega t}\right\},
\end{aligned}
$$

with a frequency $\omega>0$, the space-dependent complex-valued functions $\mathrm{E}$ and $\mathrm{H}$ solve the reduced Maxwell equations

$\operatorname{rot} E-i k H=0, \operatorname{rot} H+i k E=0$,

where the wave number $k$ is give by

$$
k^{2}=\left(\epsilon+\frac{i \sigma}{\omega}\right) \mu \omega^{2}
$$

with $\operatorname{Im} k \geq 0$.

According to Stratton and Chu [10], every solution (E, H) of the reduced Maxwell equations is divergence free and satisfies the vector Helmholtz equation.

$$
\Delta E+k^{2} E=0
$$$$
\Delta H+k^{2} H=0 .
$$

On the other hand, if $E$ solves the vector Helmholtz equation $\Delta E$ $+k^{2} E=0$ and is divergence free, then the electromagnetic Field $(E, H)$ with $\mathrm{H}:=\frac{1}{i k} \operatorname{rot} E$ solves the reduced Maxwell equations. This motivates the following boundary value problem for the vector Helmholtz equation.

Problem 1: Let $D \subset \mathrm{R}^{3}$ be a simple connected bounded domain with $C^{2}$ boundary $\partial D$. $\mathrm{D}+:=\mathrm{R}^{3} \backslash \mathrm{D}$. Let $v$ be the unit outward normal vector to $\partial \mathrm{D}$. Given the tangent field $c$ and $a$ function $\zeta$, both defined on the boundary $\partial D$, find a solution $E$ to the vector Helmholtz equation

$$
\Delta \mathrm{E}+\mathrm{k}^{2} E=0 \text {, in } D+
$$

${ }^{*}$ Corresponding author: Kuo-Ming Lee, Department of Mathematics, National Cheng Kung University, Taiwan, E-mail: kmlee@mail.ncku.edu.tw

Received September 25, 2012; Accepted September 28, 2012; Published October 01, 2012

Citation: Lee KM (2012) Boundary Integral Equations Method for the TimeHarmonic Electromagnetic Scattering. J Applied Computat Mathemat 1:e122. doi:10.4172/2168-9679.1000e122

Copyright: (c) 2012 Lee KM. This is an open-access article distributed under the terms of the Creative Commons Attribution License, which permits unrestricted use, distribution, and reproduction in any medium, provided the original author and source are credited. 
which satisfies the boundary conditions

$v \times E=c$ on $\partial D$,

$\operatorname{div} E=\zeta$ on $\partial D$,

and the radiation condition

$\operatorname{rot} \mathrm{E}(\mathrm{x}) \times \hat{x}+\hat{x} \operatorname{div} E(x)-i k E(x)=\mathrm{o}\left(\frac{1}{|x|}\right),|x| \rightarrow \infty$,

Uniformly for all directions $\hat{x}:=\frac{x}{|x|}$.

Now consider the case of an infinitely long cylinder which runs in the $x_{3}$ direction and has a cross section $\mathrm{D}$ parallel to the $x_{1} x_{2}$ plane. We have thus the following two dimensional boundary value problems for the vector Helmholtz equation.

Problem 2: $(\mathrm{H})$ Let D be a bounded simply connected domain in $\mathrm{R}^{2}$ with

$\mathrm{C}^{2}$ boundary $\Gamma:=\partial \mathrm{D} . \mathrm{D}+:=\mathrm{R}^{2} \backslash \bar{D}$. Denote $\vartheta(x)$ the unit tangent vector at

$\mathrm{x} \in \partial \mathrm{D}$ which is given by $9(\mathrm{x}):=\mathrm{e}_{3} \times v(\mathrm{x})$. Given $\xi, \rho \in \mathrm{C} 0, \alpha(\Gamma, \mathrm{C}), 0<\alpha<$ 1, find a vector field $\mathrm{E}: \bar{D} .+\rightarrow \mathrm{C}^{2}$ satisfies

1. $\mathrm{E} \in \mathrm{C}^{2}(\mathrm{D}+) \in \mathrm{C}(\bar{D}+), \operatorname{div} \mathrm{E}, \operatorname{rot} \mathrm{E} \in \mathrm{C}(\bar{D}+)$,

2. $\Delta \mathrm{E}+\mathrm{k} 2 \mathrm{E}=0$ in $\mathrm{D}+, \mathrm{k}>0$,

3. $(9, \mathrm{E})=\xi$, div $\mathrm{E}=\rho$, on $\Gamma$,

4. $\operatorname{rot} \mathrm{E}(\mathrm{x})(\mathrm{e} 3 \times \hat{x})+\operatorname{div} \mathrm{E}(\mathrm{x}) \hat{x}-\mathrm{ikE}(\mathrm{x})=\mathrm{o}\left(\left.|\mathrm{x}|\right|^{1 / 2}\right)$ for $|\mathrm{x}| \rightarrow \infty$ uniformly in all directions $\hat{x}$.

The last condition in the boundary value problem $(\mathrm{H})$ is the Sommerfeld radiation condition. It describes an outgoing wave and is essential for the uniqueness of the problem. Knauff and Kress [8] proved the unique solvability of the 3 -dimensional problem 1 . With slight modifications, their proof can also be used here to show the wellposedness of the problem (H) (See sec. 3.5 in [3] for details).

\section{Boundary Integral Equations}

Motivated by the representation theorem from stratton and Chu [10] (see also [8]), we define the solution ansatz for our problem $\mathrm{H}$.

\section{Definition (solution ansatz)}

Given $\varphi 1, \varphi 2 \in C^{0, \alpha}(\Gamma, C), 0<\alpha<1$, and a constant $\eta>0$. We call the function

$$
\begin{aligned}
& E(x):=e_{3} \times \operatorname{grad} \int_{\Gamma} \varphi 1(y) \Phi(x, y) d s(y)+i \eta \\
& \int_{\Gamma} \vartheta(y) \varphi 1(y) \Phi(x, y) d s(y)-\int_{\Gamma} v(y) \varphi 2(y) \Phi(x, y) d s(y) \\
& + \text { ingrad } \int_{\Gamma} \varphi 2(y) \Phi(x, y) d s(y),
\end{aligned}
$$

for all $\mathrm{x} \in \mathrm{D}_{+}$

the solution ansatz for the boundary value problem $\mathrm{H}$, where the function

$\Phi(\mathrm{x}, \mathrm{y}):=\frac{i}{4} H_{0}^{(1)}(\mathrm{k}|\mathrm{x}-\mathrm{y}|), \mathrm{x}, \mathrm{y} \in \mathrm{R}^{2}, \mathrm{x} \neq \mathrm{y}$,

is the fundamental solution of the scalar Helmholtz equation.

At this place, let's define the Banach space $\mathrm{C} 0, \alpha$ by

$\mathrm{C}^{0, \alpha}:=\mathrm{C}^{0, \alpha}(\Gamma, \mathrm{C}) \times \mathrm{C}^{0, \alpha}(\Gamma, \mathrm{C}), 0<\alpha<1$, and the functions $\tilde{\varphi}, \tilde{f} \in \mathrm{C} 0, \alpha$ by

$$
\varphi:=(\varphi 1, \varphi 2)^{t}, \tilde{f}:=(2 \xi, 2 \rho)^{t} .
$$

From the solution theory of the problem $\mathrm{H}$ (see [6]), we see that the solution ansatz $\mathrm{E}$ is the solution of $\mathrm{H}$ in and only if $\tilde{\varphi}$ is the solution of the following boundary integral equation (in operator form)

$$
\begin{aligned}
& (\tilde{L}+\tilde{A}) \tilde{\varphi}=\tilde{f}, \\
& \text { where } \\
& \left(\tilde{A}_{1} \varphi\right)(x):=2 \int_{\Gamma} \varphi(y) \frac{\partial \Phi(x, y)}{\partial v(x)} d s(y), \\
& \left(\tilde{A}_{2} \varphi\right)(x):=2 i \eta \int_{\Gamma}(\vartheta(x), \vartheta(y)) \varphi(y) \Phi(x, y) d s(y), \\
& \tilde{A}_{11}:=\tilde{A}_{1}+\tilde{A}_{2}, \\
& \left(\tilde{A}_{12} \varphi\right)(x):=-2 \int_{\Gamma}(\vartheta(x), v(y)) \varphi(y) \Phi(x, y) d s(y), \\
& \tilde{A}_{21}:=0, \\
& \left(\tilde{A}_{3} \varphi\right)(x):=2 \int_{\Gamma} \varphi(y) \frac{\partial \Phi(x, y)}{\partial v(x)} d s(y), \\
& \left(\tilde{A}_{4} \varphi\right)(x):=-2 i \eta k^{2} \int_{\Gamma} \varphi(y) \Phi(x, y) d s(y), \\
& \tilde{A}_{22}:=\tilde{A}_{3}+\tilde{A}_{4}, \\
& \left(\tilde{L}_{12} \varphi\right)(x):=2 i \eta\left(\vartheta(x), g r a d \int_{\Gamma}(\vartheta(y) \varphi(y) \Phi(x, y) d s(y)),\right. \\
& \left(\tilde{L}_{22} \varphi\right)(x):=-\varphi(x) \\
& \left(\tilde{L}_{11} \varphi\right)(x):=2 i \eta d i v \int_{\Gamma}(\vartheta(y) \varphi(y) \Phi(x, y) d s(y), \\
& (x)=-\varphi(x),
\end{aligned}
$$

The operator matrices $\tilde{L}, \tilde{A}: \mathrm{C}^{0, \alpha} \rightarrow \mathrm{C}^{0, \alpha}$ are defined by

$$
\tilde{L}:=\left(\begin{array}{ll}
\tilde{L}_{11} & \tilde{L}_{12} \\
\tilde{L}_{21} & \tilde{L}_{22}
\end{array}\right), \tilde{A}:=\left(\begin{array}{cc}
\tilde{A}_{11} & \tilde{A}_{12} \\
\tilde{A}_{21} & \tilde{A}_{22}
\end{array}\right) .
$$

For the numerical treatment of this problem, we need to parametrize this boundary integral equation. Write

$$
\Gamma=\{\mathrm{x}(\mathrm{t})=(\mathrm{x} 1(\mathrm{t}), \mathrm{x} 2(\mathrm{t})) \mid \mathrm{t} \in[0,2 \pi]\}
$$

for a $2 \pi$-periodic, two times continuously differentiable function $x: R \rightarrow R^{2}$.

After a lengthy mathematical computation, which will be given in some details in the appendix, the boundary integral equation (4) will be brought into the following form

$$
(\mathrm{L}+\mathrm{A}) \varphi=\mathrm{f},
$$

which is an integral equation of the second kind with an bounded invertible $\mathrm{L}$ and a compact A. 
Citation: Lee KM (2012) Boundary Integral Equations Method for the Time-Harmonic Electromagnetic Scattering. J Applied Computat Mathemat 1:e122. doi:10.4172/2168-9679.1000e122

Page 3 of 5

\section{Numerical Method}

We will apply the Nyström method to solve the boundary integral equation (5) numerically. This means that we need some quadrature rules for our integrals appear in the operators L, A. To begin with, we define the space of trigonometric functions

$$
T_{n}:=\left\{g:[0,2 \pi] \rightarrow C \mid g(t)=\sum_{k=0}^{n} a_{k} \cos k t+\sum_{k=1}^{n-1} b_{k} \sin k t, a_{k}, b_{k} \in C\right\}
$$
points

Denote by $t_{j}^{(n)}:=\frac{j \pi}{n}, \mathrm{j}=0,1, \ldots . .2 \mathrm{n}-1$, the equidistant interpolation

The interpolation operator In : C $[0,2 \pi] \rightarrow$ Tn with respect to $t_{j}^{(n)}$ is then defined by

$$
\left(I_{n} g\right)(t):=\sum_{j=0}^{2 n-1} g\left(t_{j}^{(n)}\right) L_{j}^{(n)}(t), t \in[0,2 \pi]
$$

where $L_{j}^{(n)}, \mathrm{Lj}, \mathrm{j}=0,1, \ldots, 2 \mathrm{n}-1$, is the Lagrange Basis w.r.t. $t_{j}^{(n)}$ and is given by

$$
L_{j}^{(n)}(t):=\frac{1}{2 n}\left\{1+2 \sum_{k=1}^{n-1} \cos k\left(t-t_{j}^{(n)}\right)+\cos n\left(t-t_{j}^{(n)}\right)\right\} .
$$

For the numerical solution of the boundary integral equation (5), we need to approximate the integrals. Because of the singular behavior of the Hankel functions, we have 3 different kinds of integration (see appendix). Therefore it is wise to use different quadrature rules. We use the following well-known convergent quadrature rules:

$$
\begin{aligned}
& \int_{0}^{2 \pi}\left(\cot \frac{s-t}{2}+i\right) g(t) d t \approx \int_{0}^{2 \pi}\left(\cot \frac{s-t}{2}+i\right)\left(I_{n} g\right)(t) d t, \\
& \int_{0}^{2 \pi} \ln \left(4 \sin ^{2} \frac{s-t}{2}\right) g(t) d t \approx \int_{0}^{2 \pi} \ln \left(4 \sin ^{2} \frac{s-t}{2}\right)\left(I_{n} g\right)(t) d t, \\
& \int_{0}^{2 \pi} g(t) d t \approx \int_{0}^{2 \pi}\left(I_{n} g\right)(t) d t .
\end{aligned}
$$

The quadrature (6) is used by Garrick [11], (7) is used in Martensen [12] and in Kussmaul [13]. The quadrature rule (8) is just the composite Trapezoidal rule for continuous functions. After approximating the integrals in (5) by interpolatory quadratures (6)-(8), the boundary integral equation is brought into a semidiscrete form

$$
(\operatorname{Ln}+\mathrm{An}) \varphi \mathrm{n}=\mathrm{f}, \varphi \mathrm{n}:=\varphi_{n}:=\left(\varphi_{1}^{(n)}, \varphi_{2}^{(n)}\right)^{t} \in C^{0, \alpha}
$$

We call (9) semi-discrete because it is still a functional equation. To win a full discrete system, we solve this equation at the collocation points which are the same as the interpolation points used for the trigonometric interpolation.

Mathematically, we define a projection operator Pn: $\mathrm{C}^{0, \alpha} \rightarrow \mathrm{C}^{\mathrm{n}} \times \mathrm{C}^{\mathrm{n}}$. Then the full discrete system reads

$$
\mathrm{P}_{\mathrm{n}}\left(\mathrm{L}_{\mathrm{n}}+\mathrm{A}_{\mathrm{n}}\right) \varphi_{\mathrm{n}}=\mathrm{P}_{\mathrm{n}} \mathrm{f} .
$$

We note here that the convergence $\varphi_{n} \rightarrow \varphi$ can be showed as a consequence of the convergent quadratures (6)-(8) and the interpolation. We omit the proofs which are not essential in this paper.

\section{Numerical Results}

In this section we will demonstrate the efficiency of our method through some examples. To test the advantage of the method, the parameter $\eta$ is taken to be 1 . The boundary value problem will be solved by the Nyström method with trigonometric polynomials as the underlying interpolatory space. From the asymptotic behavior of the Hankel function, we have the following representation for the far field pattern to the solution of our boundary value problem.

$$
\begin{aligned}
& E_{\infty}(\hat{x})=-\frac{e^{-i \frac{\pi}{4}}}{\sqrt{8 \pi k}}\left\{\int_{\Gamma}\left(k\left(e_{3} \times \hat{x}\right)+\eta \vartheta(y)\right) \varphi_{1}(y) e^{-i k(\hat{x}, y)} d s(y)+\right. \\
& \left.i \int_{\Gamma}(v(y)+k \eta \hat{x}) \varphi_{2}(y) e^{-i k(\hat{x}, y)} d s(y)\right\}, \hat{x} \in \Omega .
\end{aligned}
$$

\section{Example 1}

In the first example, we choose the ellipse with the parametrization $\mathrm{x}(\mathrm{t})=(\cos (\mathrm{t}), 0.5 \sin (\mathrm{t})), \mathrm{t} \in[0,2 \pi]$

For the incident field Ei, we use the plane wave mulitplicated by a constant vector

$$
\mathrm{E}^{\mathrm{i}}(\mathrm{x})=\mathrm{e}^{\mathrm{ik}(\mathrm{x}, \mathrm{d})}\left(\begin{array}{c}
1 \\
-1
\end{array}\right)
$$

with the incident direction $d=(1 / \sqrt{ } 2,1 / \sqrt{ } 2)$. The far field pattern $\mathrm{E} \infty=\left(E_{\infty}^{(1)}, E_{\infty}^{(2)}\right)$ is calculated for the two directions d and $-\mathrm{d}$. We deal with the case of a smaller wave number $(\mathrm{k}=1$, Table 1$)$ and the case of a larger wave number $(\mathrm{k}=10$, Table 2$)$. We see that the convergence is very fast in the case of an ellipse.

\begin{tabular}{|c|c|c|c|c|c|}
\hline \multirow{6}{*}{$\hat{x}=d$} & & $\operatorname{Re}\left(\tilde{E}_{\infty}^{(1)}\right)$ & $\operatorname{Re}\left(\tilde{E}_{\infty}^{(1)}\right)$ & $\operatorname{Re}\left(\tilde{E}_{\infty}^{(2)}\right)$ & $\operatorname{Re}\left(\tilde{E}_{\infty}^{(2)}\right)$ \\
\hline & 4 & -3.029115085356 & -9.059477946229 & 2.006665408697 & 9.927857754534 \\
\hline & 8 & -1.510730789273 & 0.533801178444 & 1.357562795883 & -0.343459093890 \\
\hline & 16 & -1.060418899730 & 1.484078022972 & 1.062416682157 & -1.487141012129 \\
\hline & 32 & -1.058373043098 & 1.480773361974 & 1.059156840705 & -1.484713521695 \\
\hline & 64 & -1.058373043098 & 1.480773361974 & 1.059156840705 & -1.484713521695 \\
\hline \multirow{5}{*}{$\hat{x}=-d$} & 4 & 3.495420084347 & 10.993737493727 & -2.462600064527 & -11.802651872336 \\
\hline & 8 & 0.867646086734 & 1.141495112041 & -0.895173294456 & -1.211124282675 \\
\hline & 16 & 0.478435618821 & -0.127815195366 & -0.478285303048 & 0.131479748363 \\
\hline & 32 & 0.479128007976 & -0.125769378150 & -0.478286883175 & 0.128757556023 \\
\hline & 64 & 0.479128007976 & -0.125769378150 & -0.478286883175 & 0.128757556023 \\
\hline
\end{tabular}

\section{Example 2}

In the second example, we choose a domain which is non-convex and non-symmetric. The bean-shaped domain is parametrized by $\mathrm{x}(\mathrm{t})=(\rho(\mathrm{t}) \cos (\mathrm{t}), \rho \sin (\mathrm{t})), \mathrm{t} \in[0,2 \pi]$ with the function

$\rho(t)=\frac{1+0.9 \cos (t)+0.1 \sin (t)}{1+0.75 \cos (t)}$. The incident wave Ei is taken to be the plane wave

$$
\mathrm{E}^{\mathrm{i}}(\mathrm{x})=\mathrm{e}^{\mathrm{ik}(\mathrm{x}, \mathrm{d})}\left(\begin{array}{c}
1 \\
-1
\end{array}\right)
$$

Again, we compute the two cases $\mathrm{k}=1$ and $\mathrm{k}=10$ (Table 3-4). We

$$
\begin{array}{|l|l|l|l|l|l|}
\hline \multirow{x}{*}{={ }^{n}} & n & \operatorname{Re}\left(\tilde{E}_{\infty}^{(1)}\right) & \operatorname{Re}\left(\tilde{E}_{\infty}^{(1)}\right) & \operatorname{Re}\left(\tilde{E}_{\infty}^{(2)}\right) & \operatorname{Re}\left(\tilde{E}_{\infty}^{(2)}\right) \\
\cline { 2 - 6 } & 4 & 0.034044299227 & 0.396416483233 & -0.000833096799 & -0.467877581918 \\
\hline 8 & 0.030313314173 & 0.395085099433 & -0.000833763819 & -0.466589249642 \\
\hline 16 & 0.030274526336 & 0.395082479280 & -0.000828638451 & -0.466581505068 \\
\hline 32 & 0.030274521904 & 0.395082478867 & -0.000828637801 & -0.466581504173 \\
\hline 64 & 0.030274521904 & 0.395082478867 & -0.000828637801 & -0.466581504173 \\
\hline \hat{x}=-d & 0.349416482103 & 0.617766462836 & -0.359523504381 & -0.551174747788 \\
\hline 8 & 0.344056962409 & 0.617620661423 & -0.358638819635 & -0.546837841116 \\
\hline 16 & 0.344016491614 & 0.617624533897 & -0.358632737640 & -0.546801301535 \\
\hline 32 & 0.344016487269 & 0.617624534652 & -0.358632737033 & -0.546801297405 \\
\hline 64 & 0.344016487269 & 0.617624534652 & -0.358632737033 & -0.546801297405 \\
\hline
\end{array}
$$

Table 1: Ellipse $x(t)=(\cos (t) ; 0.5 \sin (t)), k=1$. 
Citation: Lee KM (2012) Boundary Integral Equations Method for the Time-Harmonic Electromagnetic Scattering. J Applied Computat Mathemat 1:e122. doi:10.4172/2168-9679.1000e122

\begin{tabular}{|r|l|l|l|l|l|}
\hline$\hat{x}=d$ & & $\operatorname{Re}\left(\tilde{E}_{\infty}^{(1)}\right)$ & $\operatorname{Re}\left(\tilde{E}_{\infty}^{(1)}\right)$ & $\operatorname{Re}\left(\tilde{E}_{\infty}^{(2)}\right)$ & $\operatorname{Re}\left(\tilde{E}_{\infty}^{(2)}\right)$ \\
\hline 4 & -1.291299440068 & 0.343183003658 & -0.130501857685 & 0.496878648369 \\
\hline 8 & -1.294946742059 & 0.334212426346 & -0.082266685475 & 0.533403749351 \\
\hline 16 & -1.296264406645 & 0.335865209214 & -0.083020703609 & 0.534030921823 \\
\hline 32 & -1.296281335433 & 0.335681106810 & -0.083141716329 & 0.534127428988 \\
\hline 64 & -1.296281539948 & 0.335681880848 & -0.083144438344 & 0.534129034265 \\
\hline 128 & -1.296281539932 & 0.335681880731 & -0.083144437984 & 0.534129034036 \\
\hline$\hat{x}=-d$ & 4 & -1.312984822110 & 0.349319961261 & -0.029044421111 & 0.547001680965 \\
\hline 8 & -1.290880419433 & 0.392003971430 & 0.004300853221 & 0.546649067256 \\
\hline 16 & -1.292087160956 & 0.394274027179 & 0.000383682098 & 0.546888655058 \\
\hline 32 & -1.292165563090 & 0.394073662208 & 0.000311254370 & 0.546778526939 \\
\hline 64 & -1.292166390695 & 0.394073260531 & 0.000308419959 & 0.546779688645 \\
\hline 128 & -1.292166390563 & 0.394073260610 & 0.000308420282 & 0.546779688468 \\
\hline
\end{tabular}

Table 3: Bean, $x(t)=((t) \cos (t) ;(t) \sin (t)), k=1$.

\begin{tabular}{|r|l|l|l|l|l|}
\hline$\hat{x}=d$ & $n$ & $\operatorname{Re}\left(\tilde{E}_{\infty}^{(1)}\right)$ & $\operatorname{Re}\left(\tilde{E}_{\infty}^{(1)}\right)$ & $\operatorname{Re}\left(\tilde{E}_{\infty}^{(2)}\right)$ & $\operatorname{Re}\left(\tilde{E}_{\infty}^{(2)}\right)$ \\
\hline 4 & -1.497240460049 & 1.533979433764 & -1.180564001103 & 1.794007651834 \\
\hline 8 & -2.161975387064 & 1.648579446925 & -0.569287120917 & 1.028209229524 \\
\hline 16 & -2.287374827483 & 1.659888367731 & -1.435390075110 & 1.920845399020 \\
\hline 32 & -2.299962635682 & 1.667422386727 & -1.423798091589 & 1.951617424893 \\
\hline 64 & -2.299963565012 & 1.667422315500 & -1.423797950378 & 1.951616712109 \\
\hline 128 & -2.299963564915 & 1.667422315350 & -1.423797950360 & 1.951616712203 \\
\hline$\hat{x}=-d$ & 4 & -1.282601260172 & 1.325781191279 & 0.656898005281 & 2.619088118929 \\
\hline 8 & -2.250005885381 & 1.736922873984 & -2.294500984133 & 1.930082115198 \\
\hline 16 & -2.287029321533 & 1.665879681839 & -1.358715131861 & 1.899431078719 \\
\hline 32 & -2.304325580933 & 1.666620413228 & -1.311590833810 & 1.855424195000 \\
\hline 64 & -2.304326570364 & 1.666621241835 & -1.311597099786 & 1.855414728027 \\
\hline 128 & -2.304326570315 & 1.666621241626 & -1.311597098459 & 1.855414728911 \\
\hline
\end{tabular}

Table 4: Bean, $x(t)=(\rho(t) \cos (t) ; \rho(t) \sin (t)), k=10$.

note here that the convergence is because of the shape of the boundary slower than that in the first example. However, it is still satisfactory.

\section{Example 3}

In this example, we'd like to compare the method $\eta=1$ with $\eta=0$ for the stability in the case where the explicit solution of the boundary value problem is known. For this purpose we take the unit circle $\Omega$ as the domain of interest:

$$
x(t)=(\cos (t), \sin (t)), t \in[0,2 \pi] .
$$

As mentioned earlier, the solution of the boundary value is not unique if the wave number $\mathrm{k}$ is an interior eigenvalue. In the case of a unit circle, it happens to be the zeros of the Bessel functions Jn and their derivatives

$$
J_{n}^{\prime}, \mathrm{n} \in \mathrm{N} \cup\{0\} .
$$

The solution to the boundary value problem is $E(x)=H_{1}^{(1)}(k|x|)\left(e_{3} \times \frac{x}{|x|}\right)$.

The corresponding far field pattern is given by

$$
E_{\infty}(\hat{x})=\frac{-1}{\sqrt{k \pi}}(1+i)\left(e_{3} \times \hat{x}\right), \hat{x} \in \Omega \text {. }
$$

We'd like to compare the two methods on a smaller zero and a larger zero, namely

$\mathrm{z}_{1}=2.4048255576957727686 \ldots$, and $\mathrm{z}_{5}=14.9309177084877859477$ $\ldots$

We will solve the boundary value problem with those wave number k's which are close to the numbers $z_{1}$ and $z_{5}$, respectively. The results for $\eta=1$ and $\eta=0$ will then be compared. The results are listed in the Tables 5-10.

At this place, we want to draw some conclusions from our numerical results. The first thing to note is that in the case where $\mathrm{k}$ is not very close to the eigenvalue, both the modified method $\eta=1$ and the classical method $\eta=0$ converge fast (Table 5 , Table 6 , Table 8 ). In the case where $\mathrm{k}$ is close to the eigenvalue, while the modified method converges really fast regardless of the value of $\mathrm{k}$, the classical method does not converge (Table 7, Table 9, Table 10). Secondly, the modified approach is very robust in the sense that it is very stable even when the wave number $\mathrm{k}$ is numerical the same as the eigenvalue of the interior problem (Table 10).

From this we conclude that the modified method is stable for all wave numbers.

\section{Appendix}

Here we will explain the mathematical transition from (4) to (5). It is known that the method of boundary integral equations has the advantage that it reduces the dimension by one. On the other hand, it

\begin{tabular}{|l|l|l|l|l|}
\hline & \multicolumn{2}{|c|}{$\eta=1$} & \multicolumn{2}{c|}{$\eta=0$} \\
\hline$n$ & $\left.\operatorname{Re}\left(\tilde{E}_{\infty}^{(1)}\right)-E_{\infty}^{(1)}\right)$ & $\left.\operatorname{Im}\left(\tilde{E}_{\infty}^{(1)}\right)-E_{\infty}^{(1)}\right)$ & $\left.\operatorname{Re}\left(\tilde{E}_{\infty}^{(1)}\right)-E_{\infty}^{(1)}\right)$ & $\left.\operatorname{Im}\left(\tilde{E}_{\infty}^{(1)}\right)-E_{\infty}^{(1)}\right)$ \\
\hline 4 & 0.000760751082 & -0.000348248288 & -0.317896792129 & 0.776404101789 \\
\hline 8 & 0.000000000059 & -0.000000000164 & 0.000000077730 & -0.000000099322 \\
\hline 16 & 0.000000000000 & 0.000000000000 & -0.000000000000 & -0.000000000002 \\
\hline 32 & 0.000000000000 & 0.000000000000 & -0.000000000000 & -0.000000000000 \\
\hline
\end{tabular}

Table 5: $k=2: 405$

\begin{tabular}{|l|l|l|l|l|}
\hline & \multicolumn{2}{|c|}{$\eta=1$} & \multicolumn{2}{c|}{$\eta=0$} \\
\hline$n$ & $\left.\operatorname{Re}\left(\tilde{E}_{\infty}^{(1)}\right)-E_{\infty}^{(1)}\right)$ & $\left.\operatorname{Im}\left(\tilde{E}_{\infty}^{(1)}\right)-E_{\infty}^{(1)}\right)$ & $\left.\operatorname{Re}\left(\tilde{E}_{\infty}^{(1)}\right)-E_{\infty}^{(1)}\right)$ & $\left.\operatorname{Im}\left(\tilde{E}_{\infty}^{(1)}\right)-E_{\infty}^{(1)}\right)$ \\
\hline 4 & 0.000760261401 & -0.000348281565 & -0.489663840606 & 0.932861349440 \\
\hline 8 & 0.000000000059 & -0.000000000164 & -0.000024286122 & 0.000031026174 \\
\hline 16 & 0.000000000000 & 0.000000000000 & 0.000000000025 & 0.000000000572 \\
\hline 32 & 0.000000000000 & 0.000000000000 & -0.000000000120 & 0.000000000483 \\
\hline 64 & 0.000000000000 & 0.000000000000 & -0.000000000000 & -0.000000000000 \\
\hline
\end{tabular}

Table 6: $k=2: 404825$

\begin{tabular}{|l|l|l|l|l|}
\hline & \multicolumn{2}{|c|}{$\eta=1$} & \multicolumn{2}{c|}{$\eta=0$} \\
\hline$n$ & $\left.\operatorname{Re}\left(\tilde{E}_{\infty}^{(1)}\right)-E_{\infty}^{(1)}\right)$ & $\left.\operatorname{Im}\left(\tilde{E}_{\infty}^{(1)}\right)-E_{\infty}^{(1)}\right)$ & $\left.\operatorname{Re}\left(\tilde{E}_{\infty}^{(1)}\right)-E_{\infty}^{(1)}\right)$ & $\left.\operatorname{Im}\left(\tilde{E}_{\infty}^{(1)}\right)-E_{\infty}^{(1)}\right)$ \\
\hline 4 & 0.000760262961 & -0.000348281459 & -0.487096127381 & 0.934927178520 \\
\hline 8 & 0.000000000059 & -0.000000000164 & 0.331857744674 & 0.385213009420 \\
\hline 16 & 0.000000000000 & 0.000000000000 & 0.014965846216 & 0.090734474615 \\
\hline 32 & 0.000000000000 & 0.000000000000 & 0.011216877623 & 0.112044401353 \\
\hline 64 & 0.000000000000 & 0.000000000000 & 0.038544885522 & 0.126645647450 \\
\hline
\end{tabular}

Table 7: $k=2: 40482555769577$.

\begin{tabular}{|l|l|l|l|l|}
\hline & \multicolumn{2}{|c|}{$\eta=1$} & \multicolumn{2}{c|}{$\eta=0$} \\
\hline$n$ & $\left.\operatorname{Re}\left(\tilde{E}_{\infty}^{(1)}\right)-E_{\infty}^{(1)}\right)$ & $\left.\operatorname{Im}\left(\tilde{E}_{\infty}^{(1)}\right)-E_{\infty}^{(1)}\right)$ & $\left.\operatorname{Re}\left(\tilde{E}_{\infty}^{(1)}\right)-E_{\infty}^{(1)}\right)$ & $\left.\operatorname{Im}\left(\tilde{E}_{\infty}^{(1)}\right)-E_{\infty}^{(1)}\right)$ \\
\hline 4 & 0.236877497672 & 0.308692813118 & 0.271837537797 & 0.294131389369 \\
\hline 8 & 0.461665321772 & 0.622431095215 & 0.248217737580 & 0.765642734482 \\
\hline 16 & -0.001101179116 & -0.001488690955 & 0.141315007670 & 0.150142584265 \\
\hline 32 & 0.000000000000 & 0.000000000000 & 0.000000000001 & 0.000000000008 \\
\hline
\end{tabular}

Table 8: $k=14: 9309$ 
Citation: Lee KM (2012) Boundary Integral Equations Method for the Time-Harmonic Electromagnetic Scattering. J Applied Computat Mathemat 1:e122. doi:10.4172/2168-9679.1000e122

Page 5 of 5

\begin{tabular}{|l|l|l|l|l|}
\hline & \multicolumn{2}{|c|}{$\eta=1$} & \multicolumn{2}{c|}{$\eta=0$} \\
\hline$n$ & $\left.\operatorname{Re}\left(\tilde{E}_{\infty}^{(1)}\right)-E_{\infty}^{(1)}\right)$ & $\left.\operatorname{Im}\left(\tilde{E}_{\infty}^{(1)}\right)-E_{\infty}^{(1)}\right)$ & $\left.\operatorname{Re}\left(\tilde{E}_{\infty}^{(1)}\right)-E_{\infty}^{(1)}\right)$ & $\left.\operatorname{Im}\left(\tilde{E}_{\infty}^{(1)}\right)-E_{\infty}^{(1)}\right)$ \\
\hline 4 & 0.236880363951 & 0.308693042919 & 0.271840639269 & 0.294129315241 \\
\hline 8 & 0.461645963108 & 0.622403634277 & 0.248224493608 & 0.765606035841 \\
\hline 16 & -0.001100834853 & -0.001489000034 & 0.145995234827 & 0.146023296988 \\
\hline 32 & 0.000000000000 & 0.000000000000 & -0.000005622928 & -0.000011277798 \\
\hline 64 & 0.000000000000 & 0.000000000000 & -0.000003142276 & -0.000025352143 \\
\hline
\end{tabular}

Table 9: $k=14: 9309177085$.

\begin{tabular}{|l|l|l|l|l|}
\hline & \multicolumn{2}{|c|}{$\eta=1$} & \multicolumn{2}{c|}{$\eta=0$} \\
\hline$n$ & $\left.\operatorname{Re}\left(\tilde{E}_{\infty}^{(1)}\right)-E_{\infty}^{(1)}\right)$ & $\left.\operatorname{Im}\left(\tilde{E}_{\infty}^{(1)}\right)-E_{\infty}^{(1)}\right)$ & $\left.\operatorname{Re}\left(\tilde{E}_{\infty}^{(1)}\right)-E_{\infty}^{(1)}\right)$ & $\left.\operatorname{Im}\left(\tilde{E}_{\infty}^{(1)}\right)-E_{\infty}^{(1)}\right)$ \\
\hline 4 & 0.236880363949 & 0.308693042919 & 0.271840639266 & 0.294129315242 \\
\hline 8 & 0.461645963121 & 0.622403634295 & 0.248224493604 & 0.765606035866 \\
\hline 16 & -0.001100834854 & -0.001489000034 & 0.145995231681 & 0.146023299930 \\
\hline 32 & 0.000000000000 & 0.000000000000 & 0.095329722461 & -0.017433305422 \\
\hline 64 & 0.000000000000 & 0.000000000000 & 0.252437761309 & 0.103090099380 \\
\hline
\end{tabular}

Table 10: $k=14: 93091770848778594776259399738868220792$

is also known that one often has to struggle with stronger singularities. Therefore, one main point here is to split the singularities from the equations and to treat them in a unified profitable way. The singularities come from the Hankel functions $H_{0}^{(1)}, H_{0}^{(2)}$ in the fundamental solution $\Phi$ and its derivative which are buried in the solution ansatz (3). The Hankel functions has the following asymptotic behavior for $\mathrm{t} \rightarrow 0$

$$
\begin{aligned}
& H_{0}^{(1)}(t)=\frac{2 i}{\pi} \ln \frac{t}{2}+1+\frac{2 i}{\pi} C+O\left(t^{2} \ln t\right), \\
& H_{1}^{(1)}(t)=-\frac{2 i}{\pi} \frac{1}{t}+O(t \ln t) .
\end{aligned}
$$

After some cumbersome calculations, which we'd like to omit, the functional equation (4) becomes (5) with

$$
L:=\left(\begin{array}{ll}
L_{11} & L_{12} \\
L_{21} & L_{22}
\end{array}\right), A=\left(\begin{array}{ll}
A_{11} & A_{12} \\
A_{21} & A_{22}
\end{array}\right):=\left(\begin{array}{ll}
M_{11}+R_{11} & M_{12}+R_{12} \\
M_{21}+R_{21} & M_{22}+R_{22}
\end{array}\right),
$$

where

$$
\begin{aligned}
& (\mathrm{L} 11 \psi)(\mathrm{s}):=-\psi(\mathrm{s}), \\
& \left(L_{12} \psi\right)(s):=-\frac{i \eta}{2 \pi} \int_{0}^{2 \pi}\left(\cos \frac{s-t}{2}+i\right) \psi(t) d t, \\
& \left(L_{21} \psi\right)(s):=-\frac{i \eta}{2 \pi} \int_{0}^{2 \pi}\left(\cos \frac{s-t}{2}+i\right) \psi(t) d t, \\
& \left(L_{22} \psi\right)(s):=\psi(s), \\
& \text { and } \\
& \left(M_{i j} \psi\right)(s):=-\frac{1}{2 \pi} \int_{0}^{2 \pi} \ln \left(4 \sin ^{2} \frac{s-t}{2}\right) m_{i j}(s, t) \psi(t) d t, \\
& \left(R_{i j} \psi\right)(s):=\int_{0}^{2 \pi} r_{i j}(s, t) \psi(t) d t,
\end{aligned}
$$

Instead of writing down the details and all the functions, we make some remarks here:

1. The functions mij $(s, t), r_{i j}(s, t), i, j=1,2$ are as smooth as the parametrization of $\Gamma$ is (which is assumed to be of class $C^{2}$ ).
2. It can be shown that both $\ln t$ and $\ln \left(4 \sin ^{2} \frac{t}{2}\right)$ has the same singularity as $t \rightarrow 0$. Since a convergent quadrature for the latter is already at hand (7), we just split the log - singularity as we did here. The same reason is taken into account for the use of $\cot \frac{t}{2}$ instead of $\frac{1}{t}$.

3. Another worthy point of discussion about the kernel of the operators $\mathrm{L}_{12}$ and $\mathrm{L}_{21}$ is that

$$
\int_{0}^{2 \pi} \cot \frac{s-t}{2} d t=0
$$

which makes the operator L in equation (4) not invertible. Since we need $\mathrm{L}$ in equation (5) to be invertible (for the unique solvability), we rewrite the kernel as

$$
\cot \frac{s-t}{2}=\left(\cot \frac{s-t}{2}+i\right)-i
$$

The -i part is then immersed in the operators $R_{12}, R_{21}$.

Summing up, we have splitted and grouped the singularities in such a way that it is beneficial both for the theoretical treatment (unique solvability using Fredholm's theory for the 2. kind equations) and the numerical computation (convergent quadrature rules).

\section{References}

1. Kupradze WD (1956) Randwertaufgaben der Schwingungstheorie und Integralgleichungen. VEB Deutscher Verlag der Wissenschaften, Berlin.

2. Colton D and Kress R (1983) Integral Equation Methods in Scattering Theory. Wiley-Interscience Publication, New York.

3. Colton D, Kress (1998) R Inverse Acoustic and Electromagnetic Scattering Theory. (2ndedn), Springer, Berlin.

4. Werner P (1962) Randwertprobleme der mathematischen Akustik. Arch Rational Mech Anal 10: 29-66.

5. Brakhage H, Werner P (1965) Uber das Dirichletsche Au_enraumproblem four die Helmholtzsche Schwingungsgleichung. Arch Math 16: 325-329.

6. Leis R (1965) Zur Dirichletschen Randwertaufgabe des Außenraumes der Schwingungsgleichung. Mathematische Zeitschrift 90: 205-211.

7. Burton AJ, Miller GF (1971) The application of integral equation methods to the numerical solution of some exterior boundary-value problems. Proc Roy Soc London Ser A 323: 201-210.

8. Knauff W, Kress R (1979) On the exterior boundary value problem for the timeharmonic Maxwell equations. J Math Anal Appl 72: 215-235.

9. Kress R (1980) On the existence of a solution to a singular integral equation in electromagnetic reflection. J Math Anal Appl 77: 555-566.

10. Stratton JA, Chu LJ (1939) Diffraction theory of electromagnetic waves. Phys Rev 56: 99-107.

11. Garrick IE (1952) Conformal mapping in aerodynamic with emphasis on the method of successive conjugates. Nat Bur Standards Appl Math Ser 18: 137 147

12. Martensen E (1963) Uber eine Methode zum reaumlichen Neumannschen Problem mit einer Anwendung four torusartige Berandungen. Acta Math 109 $75-135$

13. Kussmaul R (1969) Ein numerisches Verfahren zur Losung des Neumannschen Au enraumproblems four die Helmholtzsche Schwingungsgleichung. Computing 4: $246-273$. 